\title{
Deliver on diagnostics to save lives
}

\author{
The release of the World Health Organization's Essential Diagnostics List highlights that diagnostics need to be \\ a fundamental part of any effective public health system and the development of rapid, reliable and reasonably \\ priced tests will be critical for tailoring appropriate treatments against infectious diseases.
}

T he adage 'an ounce of prevention is worth a pound of cure', when applied to infectious disease, highlights that more attention could be paid to developing vaccines than discovering antimicrobials. Yet this leaves out a key step entirely that of diagnostics. For example, tests that discriminate between infectious agents help clinicians select appropriate treatment, start effective therapy earlier and avoid drug overuse, which could select for resistance.

Highlighting the late recognition of diagnostics as a critical axis, the World Health Organization (WHO) recently released its first Essential Diagnostics List (EDL; https://go.nature.com/2ztcF9P), four decades after the first Essential Medicines List (EML). The EDL assesses which diagnostic tests should be readily available at various levels of health care systems; and countries should use the EDL as a starting point to curate their own diagnostic lists based on disease priorities and local factors. It is striking that 55 of the 113 tests highlighted specifically diagnose and quantify disease severity against a suite of 'priority' infectious diseases caused by viruses (human immunodeficiency virus (HIV), hepatitis B and C viruses (HBV and $\mathrm{HCV}$ ) and human papilloma virus), bacteria (tuberculosis (TB) and syphilis) and parasites (malaria and Cryptococcus), which collectively infect over 800 million and kill nearly 5 million individuals per year.

Many of these infectious disease diagnostics have been made possible due to better characterization of specific molecular markers for disease, and technological advances enabling their rapid detection with high sensitivity and in formats that do not require expensive equipment. For example, many of the rapid tests on the EDL are immunoassay-based tests that detect microbial antigens or pathogen-specific antibodies in blood, which saves significant time compared to culturing approaches, a critical bottleneck for treating patients with severe disease.

The EDL also shows that while immunoassays are attractive, sequencebased approaches (currently recommended for settings with clinical laboratories) can report on additional clinical parameters beyond pathogen presence. For example, nucleic-acid tests against several viruses (HIV, HBV, HCV) can provide quantitative information on pathogen burden and treatment efficacy, while tests targeting specific regions of the Mycobacterium tuberculosis genome can simultaneously report on TB infection and the presence of key drug resistance mutations.

The release of the EDL is to be celebrated, as it provides much needed exposure for the diagnostics field and encourages countries to adopt new tests to improve treatment outcomes. The WHO EML (https://go.nature. $\mathrm{com} / 2 \mathrm{uvW}$ xPd) helped countries prioritize and push for the use of the most useful drugs for their citizens. For example, the addition of HIV anti-retrovirals to the list in 2002 very likely contributed to the political push to make these life-saving drugs more affordable (and thus accessible) to populations in need (https://go.nature.com/2u7D6g7). It is to be hoped that the EDL may similarly increase access to diagnostics.

Some limitations remain for the practical use of diagnostic tests across different clinical settings, especially in resourcelimited point-of-care contexts. Addressing these challenges will require a balancing act between diagnostic accuracy, clinical impact and cost. For example, there is promise in using mass spectrometry and whole genome sequencing to identify pathogens and markers of drug resistance, but questions of sensitivity and specificity remain. These methods also typically require expensive equipment and technical training that is hard to access outside well-staffed reference laboratories. Approaches to diagnose disease directly from clinical samples other than blood (such as urine and faeces) will also prove of clinical relevance, as these are more accessible patient samples. Further research will also be required to either adapt existing tests or develop new approaches for emerging (and resistant) pathogens, including fungi.

Beyond scientific challenges, attention is also needed to address implementation barriers. For example, as with medicines, quality assurance and stable supply chains are critical for appropriate use of diagnostics, especially in under-resourced settings. As testing may be performed in the field, technologies to improve how data are communicated between local and national clinics will be important for real-time diagnosis and may provide avenues to incorporate the data into larger surveillance programmes that can detect emerging epidemics or inform antimicrobial stewardship efforts. Also, logistical and economic concerns remain as to how these tests can be integrated into public health programmes alongside existing tests, and how already stretched public health budgets can accommodate their cost. It is clear that political commitments are needed to expand funding to clinical laboratories at both at national and local levels, and to guarantee that tests are available to those who need them. Over the long term, by limiting the scope of treatment needed and helping halt outbreaks, implementing effective diagnostics may end up being cost neutral or save healthcare systems money.

While current diagnostics are rapidly changing the face of infectious disease treatment, much work remains to develop a suite of broadly useful tests to identify pathogens, their drug susceptibilities and inform personalized clinical decisionmaking. At Nature Microbiology we are invested in publishing advances in diagnostics-related research, whether it be new methods and devices that significantly reduce time to diagnosis; improve the sensitivity and specificity of pathogen identification (including new pathogens associated with outbreaks); enable better direct testing from clinical samples; or produce more accurate drug susceptibility prediction compared to current culturebased approaches.

The WHO EDL is an important step to better publicize the role of diagnostics in infectious disease treatment, but successful future use of diagnostics will require solid commitments to develop new tests, guarantee accurate diagnostics and to ensure that tests are accessible, affordable and used appropriately.

Published online: 25 July 2018 https://doi.org/10.1038/s41564-018-0220-9 